\title{
PAPER
}

\section{Non-convulsive status epilepticus: usefulness of clinical features in selecting patients for urgent EEG}

\author{
A M Husain, G J Horn, M P Jacobson
}

J Neurol Neurosurg Psychiatry 2003;74:189-191

Background: Non-convulsive status epilepticus (NCSE) is status epilepticus without obvious tonic-clonic activity. Patients with NCSE have altered mental state. An EEG is needed to confirm the diagnosis, but obtaining an EEG on every patient with altered mental state is not practical.

Objective: To determine whether clinical features could be used to predict which patients were more likely to be in NCSE and thus in need of an urgent EEG.

See end of article for authors' affiliations

Methods: Over a six month period, all patients for whom an urgent EEG was ordered to identify NCSE were enrolled. Neurology residents examined the patients and filled out a questionnaire without knowledge of the EEG results. The patients were divided into two groups, NCSE and non-NCSE, depending Correspondence to: Dr Aatif $M$ Husain, Box 3678, 202 Bell Building, Duke University Medical Center, Durham, NC 27710, USA aatif.husain@duke.edu

Received 29 July 2002 Accepted

1 November 2002 on the EEG result. The clinical features were compared between the two groups. The sensitivity and specificity of the features were calculated.

Results: 48 patients were enrolled, 12 in NCSE and 36 not in NCSE. Remote risk factors for seizures, severely impaired mental state, and ocular movement abnormalities were seen significantly more often in the NCSE group. The combined sensitivity of remote risk factors for seizures and ocular movement abnormalities was $100 \%$.

Conclusions: There are certain clinical features that are more likely to be present in patients in NCSE compared with other types of encephalopathy. Either remote risk factors for seizures or ocular movement abnormalities were seen in all patients in NCSE. These features may be used to select which patients should have an urgent EEG.

S atus epilepticus is a seizure that "persists for a sufficient ength of time or is repeated frequently enough that recovery between attacks does not occur."1 This definition was recently modified to "continuous seizure activity or two or more sequential seizures without full recovery lasting more than 30 minutes." ${ }^{2}$ After this 30 minute time period, there is a significant risk of neuronal injury. ${ }^{3}$ Like epileptic seizures, status epilepticus can be classified into partial and generalised varieties. ${ }^{5}$ Both have subtypes, some of which have overt convulsive activity (tonic and/or clonic) making them easy to diagnose clinically. Others lack such overt activity and are collectively referred to as non-convulsive status epilepticus (NCSE).

NCSE is an epileptic state in which there is some impairment of consciousness associated with ongoing seizure activity on EEG. ${ }^{6}$ Its classification has undergone significant evolution over the last decade and will be discussed later. NCSE is difficult to diagnose as it presents in protean ways and lacks overt convulsive activity. ${ }^{7}$ Despite the lack of convulsive activity, NCSE is thought to result in neuronal injury, ${ }^{3}{ }^{8}$ and so its recognition and treatment are critical.

Although there are several reports of clinical features of NCSE, ${ }^{5-19}$ there have been no studies evaluating the presence of these features prospectively in a series of patients in NCSE. The definitive diagnosis is made with an EEG; however, it is often not possible to obtain an urgent EEG in every patient with an altered mental state. Our aim in this study was to evaluate whether clinical features can help predict which patients have a high probability of being in NCSE and thus should undergo an urgent EEG.

\section{METHODS}

The study was conducted over a six month period at a teaching hospital. As paediatric patients are not admitted to this hospital, only adults (age 15 years or older) were included. All patients for whom an urgent EEG was requested to rule out NCSE were enrolled. In line with hospital policy, only neurology residents could order urgent EEGs. Thus a neurology resident evaluated all patients for whom such a study was ordered. After evaluating the patient, the resident was asked to complete the study questionnaire.

The questionnaire was designed to elicit details of the patient's history and examination in a structured format. A summary of the questions asked is presented in table 1 . These questions were based on features of NCSE in published reports. ${ }^{20}$ Among recent risk factors for status epilepticus specifically asked about were acute intracranial events, drug intoxication or withdrawal, and antiepileptic drug noncompliance. Remote risk factors asked about included previous stroke, tumour, previous neurosurgery, dementia, and meningitis. Mental status testing was done using the Glasgow coma scale (GCS). Ocular movement abnormalities included nystagmus, hippus, and sustained eye deviation in any direction. Subtle motor activity included not only persistent muscle twitches in the extremities or face but also automatisms. Abnormal laboratory values were also noted on the questionnaire.

The neurology residents were asked to complete the questionnaire after evaluating the patient and before completion of the EEG. An American Board of Clinical Neurophysiology certified electroencephalographer interpreted the EEG without knowledge of the clinical assessment. NCSE was diagnosed if the EEG was thought to be consistent with electrographic status epilepticus. As the focus of this study was diagnosis and not treatment, the patients thought to be in absence status epilepticus (ASE) were not separated from those in complex partial status epilepticus (CPSE).

\section{Statistical analysis}

On the basis of the EEG, patients were subclassified into an NCSE group and a non-NCSE group. The duration of symptoms

Abbreviations: ASE, absence status epilepticus; CPSE, complex partial status epilepticus; GCS, Glasgow coma scale; NCSE, non-convulsive status epilepticus 


\section{Table 1 Questions asked}

Duration of symptoms

Recent risk factors for seizures

Remote risk factors for seizures

Tonic-clonic activity in current episode

History of epilepsy

Mental state/Glasgow coma scale

Ocular movement abnormalities

Subtle motor activity (face and extremities)

\begin{tabular}{llll} 
Table 2 & \multicolumn{3}{l}{ Comparison of NCSE and non-NCSE groups } \\
\hline & $\begin{array}{l}\text { NCSE } \\
(\mathbf{n}=12)\end{array}$ & $\begin{array}{l}\text { Non-NCSE } \\
(\mathbf{n}=36)\end{array}$ & p Value \\
\hline Recent risk factors & $9(75)$ & $26(72)$ & NS \\
Remote risk factors & $9(75)$ & $15(42)$ & 0.05 \\
Tonic clonic activity & $6(50)$ & $16(42)$ & NS \\
History of epilepsy & $2(17)$ & $10(28)$ & NS \\
$\begin{array}{l}\text { Glasgow coma scale score } \\
\text { Ocular movement }\end{array}$ & $6(50)$ & $16(44)$ & 0.0002 \\
$\begin{array}{l}\text { abnormalities } \\
\text { Subtle motor activity }\end{array}$ & $9(75)$ & $20(56)$ & NS \\
\hline
\end{tabular}

Values are $\mathrm{n}(\%)$.

NCSE, non-convulsive status epilepticus.

in the two groups was compared using Student's $t$ test. The frequency of each of the clinical features noted in table 1 was determined in both groups. Two tailed $\chi^{2}$ analyses were used to compare the frequency of the clinical features between the two groups. The GCS scores were compared between the two groups using Student's $t$ test. The sensitivity and specificity of each of these features for NCSE were also calculated.

\section{RESULTS}

Forty eight patients were enrolled, 12 in the NCSE group and 36 in the non-NCSE group. All 12 patients in the NCSE group had CPSE; none had ASE. Mean age was 59.4 years (range 34 to 89) in the NCSE group and 55.6 (range 15 to 88) in the non-NCSE group. The female to male ratio was $8: 4$ in the NCSE group and 16:20 in the non-NCSE group. The mean duration of symptoms was 19.8 hours for the NCSE group and 48.0 hours for the non-NCSE, but this difference was not statistically significant.

The frequency of the clinical features in the two groups is presented in table 2 . Results of the $\chi^{2}$ analyses between the groups are also presented in the table. Remote risk factors for seizures, a depressed mental state (mean score of 4.5 on the GCS), and ocular movement abnormalities were significantly more common in patients in the NCSE group. Recent risk factors for seizures, the presence of tonic-clonic activity, a history of epilepsy, and subtle motor activity were not significantly different between the groups.

The sensitivity and specificity of each of the clinical features noted above are presented in table 3 . Neither the sensitivity nor the specificity was greater than $80 \%$ for any of the clinical features. The specificity of ocular movement abnormalities was high (86\%), but its sensitivity was low (50\%). However, the combined sensitivity (presence of either/or) of remote risk factors for seizures and ocular movement abnormalities was $100 \%$, though the specificity was only $55 \%$. No two other features had a combined sensitivity of $100 \%$.

A review of the laboratory data showed that seven of the 12 patients in the NCSE group (58\%) and 19 of the 36 patients in the non-NCSE group (53\%) had abnormal laboratory results (NS by two tailed $\chi^{2}$ analysis). The most common abnormalities included a raised blood urea nitrogen to creatinine ratio
Table 3 Sensitivity, specificity, positive predictive value, and negative predictive value for NCSE

\begin{tabular}{lll}
\hline & Sensitivity (\%) & Specificity (\%) \\
\hline Recent risk factors & 75 & 28 \\
Remote risk factors & 75 & 58 \\
Tonic clonic activity & 50 & 58 \\
History of epilepsy & 17 & 72 \\
Ocular movement abnormalities & 50 & 86 \\
Subtle motor activity & 75 & 44 \\
\hline NCSE, non-convulsive status epilepticus.
\end{tabular}

(14 patients), a raised white blood cell count ( 14 patients), and raised serum glucose (nine patients).

\section{DISCUSSION}

Convulsive status epilepticus is easy to diagnose by virtue of its obvious clinical manifestations. Though it can cause neuronal damage, ${ }^{321}$ the straightforward clinical diagnosis leads to early treatment. As its name implies, NCSE lacks the convulsions typically seen in status epilepticus; however it, too, can cause neuronal injury. ${ }^{38}$ It can have protean symptoms associated with an alteration of mentation which makes it difficult to diagnose clinically. It is thus often missed in patients presenting with an altered mental state. ${ }^{7}$ An EEG is needed to confirm the diagnosis. Obtaining an urgent EEG in every patient presenting with an altered mental state is impractical, especially after regular business hours when technologists are not easily available. This study was designed to determine whether symptoms and signs could be identified that would be indicative of NCSE, allowing a more selective approach to ordering urgent EEGs.

The classification of NCSE has undergone evolution over the last decade. NCSE has long been divided into two main categories: absence status epilepticus (ASE) and complex partial status epilepticus (CPSE). ${ }^{16} 2223$ Until recently most cases of NCSE were considered ASE, and CPSE was thought to be a rarity. ${ }^{1622} 24$ Several investigators have, however, shown that CPSE is more common than previously thought $t^{102325}$ and that many cases of ASE are actually cases of CPSE that have generalised. ${ }^{26}{ }^{27}$ Tomson et al reported on 32 consecutive NCSE patients, most of whom had CPSE, and suggested that in adults most cases of NCSE are in fact partial in onset. ${ }^{26}$ Our results support those findings, with all cases in the NCSE group being CPSE. The distinction between ASE and CPSE is an important one, as ASE is usually easier to treat and may not be associated with neuronal damage. Regardless of the classification system used, subclassification of NCSE is difficult because clinical and EEG characteristics often overlap, so urgent treatment should be considered.

The clinical symptoms of NCSE are protean. They are reviewed in detail elsewhere ${ }^{20}$ and only summarised here. The only symptom seen consistently is an alteration in mental state. This can range from mild confusion to profound impairment of consciousness. At times there can be fluctuation in the degree of impairment. ${ }^{28}$ Though psychomotor retardation is most common, agitation and excitation can also occur. Tonic and/or clonic activity can be seen in any extremity. Head and eye deviation, hippus, and nystagmoid eye jerks have been described. Focal myoclonic jerks involving the face, eyelids, or extremities have also been noted, more commonly with ASE. ${ }^{20}$ Automatisms may also occur and include lip smacking, orofacial movements, or hand/arm movements. Various language and cognitive difficultiesincluding aphasia, perseverations, echolalia, confabulations, and so on-have also been noted. Autonomic disturbances, such as flatulence, borborygmi, and belching, have occurred in a few patients. Sensory and psychic phenomena have also 
been reported but are much less common. ${ }^{20}$ However, none of these symptoms and signs has been evaluated in a prospective study to see how often they occur in NCSE and how often they occur in other encephalopathic patients. If certain clinical features can be shown to be associated with NCSE, the selection of patients for urgent EEG can be made more accurate and cost-effective.

At many centres an urgent EEG to exclude NCSE is ordered when there is unexplained alteration in mental state and definite suspicion of NCSE based on the presence of certain clinical features (for example, ongoing clonic activity, nystagmus, or eye deviation). Though these clinical features have been associated with NCSE in retrospective studies, ${ }^{12-15}$ they have not been evaluated in a prospective manner. Retrospective reviews of clinical features of NCSE cannot be used to determine the frequency of the relevant clinical features in patients who are encephalopathic but not in NCSE. Our present study was designed to overcome that bias by evaluating all patients suspected of being in NCSE before obtaining an EEG.

In this study women outnumbered men in the NCSE group by two to one. Men were more common in the non-NCSE group. Others have also noted a female preponderance in NCSE. ${ }^{29}$ Remote risk factors were seen more often in the NCSE group than the non-NCSE group $(75 \% \vee 42 \%, \mathrm{p}=0.05)$ : these included conditions such as previous stroke, neurosurgical intervention, brain tumour, history of meningitis, and so on. NCSE patients had lower GCS scores $(4.5 v 9.2, \mathrm{p}<0.001)$ and more often had ocular movement abnormalities (50\% v $14 \%$, $\mathrm{p}<0.05)$. Nystagmoid eye jerks, hippus, repeated blinking, and persistent eye deviation were considered to be eye movement abnormalities; slow roving eye movements were not included in this group. It is important to note that the occurrence of certain findings often associated with NCSE (that is, recent risk factors for seizures, a history of tonic-clonic activity recently, a history of epilepsy, and subtle motor activity) was not significantly different between the two groups. Also abnormal laboratory tests were just as likely to be found in either group.

None of the clinical features evaluated had a high sensitivity and specificity individually. However, the combined sensitivity for "remote risk factors for seizures" and "eye movement abnormalities" was $100 \%$. Though the specificity of these two criteria for correctly diagnosing NCSE was low, it is the sensitivity that is more important for screening patients for urgent EEGs. The presence of either of these two features can help identify patients who need an urgent EEG. No two other clinical features had a diagnostic sensitivity of $100 \%$ for NCSE. Recent risk factors for seizures, a history of tonic-clonic activity recently, a history of epilepsy, and subtle motor activity-all previously thought to be suggestive of NCSE-had neither high sensitivity nor high specificity for NCSE.

It should be noted that in this study all patients were evaluated by a neurology resident and thus underwent a careful neurological history and examination. Additionally the resident considered all ancillary tests to determine whether or not an urgent EEG should be ordered. This should be considered when generalising these finding to other settings.

In this study various symptoms and signs thought to be commonly associated with NCSE were evaluated prospectively to see if they could be used to diagnose this condition. Remote risk factors for seizures, a low GCS score, and ocular movement abnormalities were found more often in patients with NCSE. The presence of remote risk factors for seizures, ocular movement abnormalities, or both had a diagnostic sensitivity of $100 \%$. Other clinical findings commonly thought to be associated with NCSE (such as recent risk factors for seizures, tonic-clonic activity recently, a history of epilepsy, and abnormal motor activity) were seen just as often in patients with an encephalopathy other than NCSE. Our findings may help to prioritise patients for urgent EEGs. It should be noted, however, that not all patients in NCSE had these findings, and these clinical features cannot serve as a substitute for an EEG in making the diagnosis.

\section{ACKNOWLEDGEMENTS}

Study supported by a house staff research grant, Medical College of Pennsylvania. Assistance provided by neurology residents of the Medical College of Pennsylvania is gratefully acknowledged.

\section{Authors' affiliations}

A $M$ Husain, Department of Medicine (Neurology), Duke University, Durham, North Carolina, USA

G J Horn, Department of Medical Psychology, Florida Hospital, Orlando, Florida, USA

M P Jacobson, Department of Neurology, Temple University, Philadelphia, Pennsylvania, USA

Competing interests: none declared

\section{REFERENCES}

1 Commission on Classification and Terminology. International League Against Epilepsy. Proposal for revised clinical electroencephalographic classification of epileptic seizures. Epilepsia 1981;22:489-501.

2 Epilepsy Foundation. Treatment of convulsive status epilepticus: recommendations of the Epilepsy Foundation of America's working group on status epilepticus. JAMA 1993:270:854-9.

3 Fountain NB, Lothman EW. Pathophysiology of status epilepticus. J Clin Neurophysiol 1995;12:326-42.

4 Gilliam FG. Status epilepticus. In: Wyllie E, ed. The treatment of epilepsy: principles and practice, $2^{\text {nd }}$ ed. Baltimore: Williams and Wilkins, 1996:666-76.

5 Treiman DM. Electroclinical features of status epilepticus. J Clin Neurophysiol 1995;12:343-62

6 Kaplan PW. Nonconvulsive status epilepticus. Semin Neurol 1996;16:33-40.

7 Drislane FW. Presentation, evaluation, and treatment of nonconvulsive status epilepticus. Epilepsy Behav 2000;1:301-14

8 Meldrum BS, Vigouroux RA, Brierley JB. Systemic factors and epileptic brain damage. Arch Neurol 1973;29:82-7.

9 Aminoff MJ, Simon RP. Status epilepticus: causes, clinical features and consequences in 98 patients. Am J Med 1980;69:657-66.

10 Ballenger CE, King DW, Gallagher BB. Partial complex status epilepticus. Neurology 1983;33:1545-52.

11 Treiman DM, De Giorgio CMA, Salisbury SM, et al. Subtle generalized convulsive status epilepticus [abstract]. Epilepsia 1984;25:653.

12 Kaplan PW. Nonconvulsive status epilepticus in the emergency room. Epilepsia 1996;37:643-50

13 Lowenstein DH, Aminoff M. Clinical and EEG features of status epilepticus in comatose patients. Neurology 1992;42:100-4.

14 Guberman A, Cantu-Reyna G, Stuss D, et al. Nonconvulsive generalized status epilepticus: clinical features, neurophysiological testing, and long-term follow-up. Neurology 1986;36:1284-91.

15 Drislane FW, Schomer DL. Clinical implications of generalized electrographic status epilepticus. Epilepsy Res 1994;19:111-21.

6 Celesia GG. Modern concepts of status epilepticus. JAMA 1976;235:1571-4.

17 Tomson T, Svanborg E, Wedlund J-E. Nonconvulsive status epilepticus: high incidence of complex partial status. Epilepsia 1986;27:276-85.

18 DeLorenzo RJ, Waterhouse EJ, Towne AR, et al. Persistent nonconvulsive status epilepticus after the control of convulsive status epilepticus. Epilepsia 1998;39:833-40.

19 Drislane FW, Blum AS, Schomer DL. Focal status epilepticus: clinical features and significance of different EEG patterns. Epilepsia 1999:40: 1254-60

20 Shorvon S. Clinical forms of status epilepticus. In: Shorvon S, ed. Status epilepticus: Its clinical features and treatment in children and adults. Cambridge: University Press, 1994:34-138

21 Wasterlain CG, Fujikawa DG, Penix L, et al. Pathophysiological mechanisms of brain damage from status epilepticus. Epilepsia 1993;34(suppl 1):S37-53

22 Jagoda A, Riggio S. Nonconvulsive status epilepticus in adults. Am J Emerg Med 1988;6:250-4.

23 Mayeux R, Lueders $\mathrm{H}$. Complex partial status epilepticus: case report and proposal for diagnostic criteria. Neurology 1978;28:957-61.

24 Gastaut H. Classification of status epilepticus. In: Delgado-Escueta AV Wasterlain CG, Treiman DM, et al, eds. Status epilepticus. Adv Neurol 1983;34:15-35.

25 Hauser WA. Status epilepticus: frequency, etiology, and neurological sequelae. In: Delgado-Escueta AV, Wasterlain CG, Treiman DM, et al, eds. Status epilepticus. Adv Neurol 1983;34:3-14.

26 Tomson T, Lindbom U, Nilsson BY. Nonconvulsive status epilepticus in adults: thirty-two consecutive patients from a general hospital population. Epilepsia 1992;35:829-35

27 Treiman DM. Generalized convulsive status epilepticus in the adult. Epilepsia 1993;34(suppl 1):S2-11

28 Treiman DM, Delgado-Escueta AV. Complex partial status epilepticus. In: Delgado-Escueta AV, Wasterlain CG, Treiman DM, et al, eds. Status epilepticus. Adv Neurol 1983;34:69-81.

29 Von Rossum J, Groeneveld-Ockhuysen AAW, Arts RJHM. Psychomotor status. Arch Neurol 1985;42:989-93 